\title{
INFLUENCE OF CHLORIDE CORROSION ON THE STRESS-STRAIN STATE OF THE REINFORCED CONCRETE PLATE ON AN ELASTIC BASE
}

\begin{abstract}
Alexandr SHIMANOVSKY, Department of Technical Physics and Engineering Mechanics, Faculty of Mechanical Engineering, Belarusian State University of Transport, Kirova Str. 34, Gomel, 246653, Belarus. tm.belsut@ gmail.com (corresponding author) Sergey GRIDNEV, Department of Structural Mechanics, Institute of Civil Engineering, Voronezh State University of Architecture and Civil Engineering, Address: 20-letiya Oktayabrya Str., 84, Voronezh, 394006, Russia; Fluid Dynamics and Seismics Lab, Moscow Institute of Physics and Technology (State University), Institutskiy per. 9, Dolgoprudny, Moscow Region, 141701, Russia. gridnevs y@rambler.ru

Igor OVCHINNIKOV, Department of Car Roads and Bridges, Car and Road Building Faculty, Perm National Research Polytechnic University, Akademika Koroleva Str. 19a, Perm, 614013, Russia. bridgesar@mail.ru

The operating conditions of a number of reinforced concrete structures used in agricultural production cause the need of taking into account the chloride corrosion to analyze their long-term operation strength. Under the influence of chloride corrosion, the destruction of concrete and corrosion of metal reinforcement appear and lead to a change in the stress-strain state of the reinforced structures and to their durability reduction. In the presented work, the mathematical model of the elastically based reinforced concrete plate under the influence of chloride corrosion is presented as a fourth-order nonlinear differential equation in partial derivatives. The effect of concrete degradation on the stress-strain state of the structure was implemented by a technique involving a method of successive parameter perturbations to solve the nonlinear part of the problem and the grid method for solving the boundary part of the problem. There was developed a program that allows to analyze the behavior of the reinforced plate under the action of chloride corrosion on one or both surfaces of the plate. The algorithm of the program allows to change the boundary conditions, reinforcement parameters, direction, concentration and chlorides' penetration velocity. There was analyzed the change in the stress-strain state of the reinforced plate over time for one of the possible variants of chloride corrosion pattern. On the basis of the computational results' analysis it was demonstrated how the durability curve changes due to the action of chloride corrosion.
\end{abstract}

Keywords: chloride corrosion, durability, reinforced concrete plate, stress-strain state

\section{INTRODUCTION}

Reinforced concrete structures are widely used in agricultural production (Šadzevičius, 2015). However, one of the shortcomings of the structures with metal reinforcement is their weak resistance to chloride corrosion. The chloride medium reaches the surface of the reinforcement due to penetrating into the protective layer concrete. After the critical concentration of chlorides at the reinforcement surface is exceeded its corrosion starts. The corrosion can occur evenly and unevenly around the reinforcement perimeter, but in both cases it causes the decrease in the cross-sectional area size, the loss of adhesion of concrete to the reinforcement, the appearance and growth of cracks, incorrect operation of the reinforcement-concrete interaction. As a result, the stress-strain state of the reinforced structure changes, its durability reduces (Zhou, 2015; Neville, 1995; Alekseev, 1990). The kinetics of the stress state change and the general reinforced concrete destruction are affected by the nature and level of loading, the peculiarities of chloride-containing medium contact with the reinforced structure surface, the chlorides' concentration and other parameters (Parant, 2007; Chen, 2008).

Usually, to perform the calculation of reinforced structural elements it is used a methodology based on the limiting states analysis and the reinforced structure is considered to be destroyed if the external load forces exceed the load-bearing capacity of the structure determined using the limit characteristics of the reinforced concrete components. However, while operation of the reinforced structures the reinforced concrete, in overwhelming majority of cases, is destroyed not from the load increase to a dangerous level, but from a significant deterioration of the concrete properties and the reinforcement crosssection reduce so that the structure is destroyed by the workload. Therefore, the deformation approach (Karpenko, 1996) is more correct for the calculation of the reinforced structures behavior in the contact with a chloride-containing medium.

Most of the existing calculating methods for the reinforced structures under to chlorides action are based on the limiting states method adjustment in the direction of taking into account the effects of the aggressive environment, but it is not correct from the point of view of the deformation theory.

Copyright (C) 2017 The Authors. Published by Aleksandras Stulginskis University. This is an open-access article distributed under the terms of the Creative Commons Attribution License (CC-BY 4.0), which permits unrestricted use, distribution, and reproduction in any medium, provided the original author and source are credited. 
In due time A.F. Polak et al. (Polak, 1983; Müller, 1985; Wright, 1985) developed the methods for mathematical modeling of the reinforced structures behavior in chemical manufacturing environments and various ways of their durability prediction. D.N. Frangopol (Frangopol, 1997; Kong, 2002; Biondini, 2004; Frangopol, 2004) paid special attention to taking into account the processes probabilistic nature of the deformation and the corrosion destruction. E.A. Guzeev et al. (Guzeev, 1984) suggested the models of the force and environmental (i.e., caused by the action of corrosive media) destruction of the reinforced elements and they observed a strong influence of the action of both the corrosive media and the force factors on the stress-strain state of reinforced structures.

A.I. Popesko (Popesko, 1996) developed a calculation technique for the corroding reinforced concrete structures, based on the use of integral characteristics of the structure deformative properties. V.I. Solomatov and V.P. Selyaev (Solomatov, 1987) proposed to use the special degradation functions of rigidity and load-bearing capacity for the calculation of concrete cross sections under conditions of corrosive media action. In paper (Saetta, 1999) it was introduced a special function-damage from the aggressive media action for modeling the process of reinforced structures deformation in an aggressive media.

V.V. Petrov and I.G. Ovchinnikov (Petrov, 1987; Ovchinnikov, 2002; Ovchinnikov, 2014) proposed to use a special approach to the structures analysis in an aggressive media based on the usage of system of models reflecting different aspects of the deformation process and destruction of the reinforced structural element. The model of the analyzed construction interacting with the aggressive media is represented as a totality of models: the model of the constructive element, the model of the ground base, the material model, the media impact model, the model of the reached limit state.

The purpose of the presented work is to develop a methodology for analysis of reinforced concrete elements taking into account the special properties of the material and the reinforcement, and also the destructive effect of chloridecontaining media.

\section{ANALYSIS METHOD}

In the work (Ovchinnikov, 2012), it was considered the problem of creation the relations for the deformation model of a reinforced plate on an elastic base under the action of chloride corrosion; a nonlinear resolving equation was presented:

$$
\left(\frac{\partial^{2}}{\partial x^{2}}\left[I_{11} \frac{\partial^{2} W}{\partial x^{2}}\right]+\frac{\partial^{2}}{\partial x^{2}}\left[I_{12} \frac{\partial^{2} W}{\partial y^{2}}\right]+2 \frac{\partial^{2}}{\partial x \partial y}\left[I_{3} \frac{\partial^{2} W}{\partial x \partial y}\right]+\frac{\partial^{2}}{\partial y^{2}}\left[I_{21} \frac{\partial^{2} W}{\partial x^{2}}\right]+\frac{\partial^{2}}{\partial y^{2}}\left[I_{22} \frac{\partial^{2} W}{\partial y^{2}}\right]\right)-2 m \nabla^{2} W+n W=p(x, y)
$$

In this equation: $W$ - the deflection of the plate, measured from the median plane; $I_{11}, I_{12}, I_{3}, I_{21}, I_{22}-$ stiffness characteristics - definite integrals over the plate thickness from complex deflection functions, concentration of chloridecontaining medium, parameters of concrete and reinforcement; $m, n$-elastic base characteristics; $p(x, y)-$ external load intensity.

The distribution law of the medium chloride over volume of the reinforced plate is found from the equation:

$$
\frac{\partial C}{\partial t}=\operatorname{div}(D \cdot \operatorname{grad} C)-g(C)
$$

where $C$ - the concentration of chlorides at the plate volume point, $t$ - time; $D$ - diffusion coefficient; $g(C)$ chloride binding rate. From the equation of damages accumulation it is found the distribution law for the damage parameter over the plate volume to the considered moment of time, and the area of the corroded reinforcement is determined by the means of the corrosion wear equation. To obtain the final result, the equation (1) should be solved for each time point taking into account the stiffness characteristics changes affected by the mentioned factors.

The problem of a physically nonlinear plate calculating is divided into two parts: the solution of the nonlinear part of the problem (because of the concrete deformation diagram nonlinearity, the influence of chlorides and the reinforcement corrosion) and the solution of its boundary-value part.

In our case, the equation (1) was solved by combining the method of successive parameter perturbations to solve the problem nonlinear part and the grid method for solving the boundary-value part of the problem.

For the general case of the chlorides action on a reinforced plate, it is difficult to obtain the equation (2) solution.

Therefore, if the chlorides act the plate from one or both sides and the distribution of chlorides over the surface is fairly uniform, it can be assumed that there is the one-dimensional diffusion process in a direction perpendicular to the plate plane. In the case of the one-dimensional diffusion process, the diagram of the chloride concentration distribution over the plate thickness is a rectangular triangle, so it is allowed us to use the following function for the chloride concentration field approximation:

$$
C(x, t)=C_{0}\left[1 \pm \frac{z}{L(t)}-\frac{h}{2 L(t)}\right]
$$

where: $z$ - the point coordinate along the plate thickness; $t$-time; $C_{0}$ - the chlorides concentration value on the plate surface; $h$ - the plate thickness; $L(t)$ - the motion equation of the boundary of the triangular fuzzy front of the chloride concentration over the plate thickness (Ovchinnikov, 2007): 


$$
L(t)=\alpha t^{n}
$$

where $\alpha, n$ - coefficients.

To take into account the influence of the stressed state form on the chlorides penetration into the plate the following equation can be used:

$$
L_{j}(t)=\alpha_{j} t^{n_{j}}
$$

where $j=1$ for the stretched plate section zone, $j=2$ - for the compressed one.

The coefficient $\alpha$ value can be different for different contact points of the circuit and the chloride-containing medium. If $\alpha=0$, it means that the considered part of the circuit is protected from the aggressive medium action, if $\alpha>0$ - the chloride corrosion occurs. The performed numerical analysis has shown that the function (3) description of the chlorides concentration field distribution along the plate thickness is quite correctly.

To calculate the reinforced plate, it is possible to consider various programs of the plate loading and the effects of chlorides on its surface. It was assumed that the interaction of chlorides with the reinforced plate can be represented by the following sequenced steps.

1. The plate is loaded without chlorides action. At the same time the stress state is formed only from the action of the plate load.

2. The resulting stressed state causes the accumulation of damages and changes in the stress-strain state of the plate due to the influence of damages on the deformation process. At the end of this stage $\left(t=t_{0}\right)$ a certain level of plate stresses and damages is achieved.

3. The boundary conditions of the chlorides action model are formulated. It means that it is assumed that the chlorides concentration on the surface increases from 0 to $C_{0}$ over a certain time interval $t_{b}$.

4. The chlorides penetration into the plate and the degradation of the concrete starts. The stress-deformed and damaged state changes due to the action of the applied load, chlorides and the damages accumulation. The stage continues until the critical value of the chlorides concentration at the reinforcement location $C_{c r}$ is achieved.

5. At this stage, the process of concrete degradation continues, its mechanical characteristics change, the damages accumulation and the reinforcement corrosion deterioration take place. The change in the stress-strain state is caused by the coupled action of the load, the concrete degradation, the reinforcement corrosion, the concrete damages accumulation.

6. The limiting state of any kind occurs: reaching the damage ultimate level of $(L=1)$ at any point of the plate; the achievement of the maximum stress level caused by the reinforcement cross-section area decrease.

To solve the fourth-order equation (1) in partial differentials, the method of finite differences is applied. This method has proved itself well for the calculating of the variable thickness plates when the plate bending equation is outwardly similar to equation (1).

For the solution of the fourth-order equation (1) in partial differential, the method of finite differences is used. This method has proved itself well for the calculating of plates of variable thickness when their bending equation is outwardly similar to the equation (1).

The volume of the calculated reinforced plate is covered by a three-dimensional rectangular grid of nodes in an amount of $M+1$ along the $O x$ axis direction; $N+1-$ along $O y$ direction; $K+1-$ along $O z$ direction. To use symmetric difference operators for the boundary plate points, it is added one more row of nodes external to the plate along the directions $O x$ and $O y$ is added. In this case, a certain increase in the number of nodes is compensated by a decrease in the calculation error by several times.

The layer of the middle plate surface nodes is used to write the equation (1) in the difference form, and the derivatives in the differential operator of the equation are replaced by difference formulas. Since derivatives of the fourth and second orders can be observed in the differential operator, the use of these difference formulas of the second accuracy order gives a difference template showing that the difference analogue of the differential operator $D(W)$ of the considered problem written for the node $(i, j)$ is nothing more than a linear combination of 13 neighboring nodal values of deflection.

Using this template, the difference operator can be written for each inner node of the grid middle layer, i.e. node that does not lie on the boundary and it is not external. In this case, the required values are the nodal values of the middle surface deflections $W_{i j}, i=1, \ldots, M+3, j=1, \ldots, N+3$. To find these quantities a system of linear algebraic equations (SLAE) should be applied:

$$
A \bar{W}=B,
$$

where $A-$ a square matrix consisting of $(M+3)(N+3)$ rows and $(M+3)(N+3)$ columns, each row of the matrix contains the coefficients of the difference operator for one of the nodes $(i, j)$ of the medium grid layer; $B-\mathrm{a}$ column of free terms containing the summand-constants of the corresponding equations of differences; $\bar{W}-$ vector of unknown deflections.

To fill the SLAE matrix, it is necessary to know the node stiffness values $I_{11}, I_{12}, I_{21}, I_{22}, I_{3}$, their calculation is the integrals calculation over the thickness of the plate from the functions found in the previous step. Integrals over the thickness of the plate are found by the Simpson method for equidistant nodes. The stretched zones of the plate are separated from the compressed by the neutral surface $z=z_{0}$. Therefore, to calculate the stiffness values it is necessary to 
set the values of $z_{0}$ for each $(i, j)$ node of the middle layer, and to point the location of the compressed zone - above $(s>0)$ or lower $(s<0)$ of the neutral level:

$$
z_{0}=-\frac{\varepsilon_{x}+\varepsilon_{y}}{\chi_{x}+\chi_{y}} ; s=\operatorname{sign}\left(\chi_{x}+\chi_{y}\right) \text {. }
$$

If the $z_{0}$ and $s$, are known it is possible to calculate rigidities, fill the SLAE matrix $A$ and the vector $B$ and calculate the deflections $W_{i j}$. If the deflections are calculated the curvatures and deformations for the new values of $z_{0}$ and $s$ can be obtained. Consequently, the plate calculating problem is solved iteratively. Preliminary calculations showed that the application of the simple iteration method provides good convergence: in the case of rigid clamping the convergence is achieved in 6-7 iterations with an error of $10^{-4}$, and in the case of hinged support it can be achieved in 3 iterations.

\section{RESULTS AND DISCUSSION}

Using the developed program there was performed an analysis of the stress-strain state and evaluation of the durability of the loaded reinforced plate on an elastic base under the action of chloride corrosion. It was assumed that the load is statically applied to the plate and that it is evenly distributed along the middle plane of the plate. The following cases of the chlorides action on the plate surface were investigated: at first the chlorides act only on the entire upper surface, then only on the lower surface and finally both on the lower and upper surface; at the same time the end faces of the plate are isolated from the chlorides action.

Various schemes for plate supporting were considered:

a) hinged support along the contour, b) rigid pinching along the contour, c) the two opposite sides are hinged, and the other two opposite sides are rigidly clamped, d) the two adjacent sides are hinged, and the other two adjacent sides are rigidly clamped.

Plate dimensions, reinforcement parameters, mechanical characteristics of the concrete and reinforcement, the load intensity, the characteristics of the elastic base and the aggressive medium are described below.

The geometric dimensions of the reinforced plate: thickness $h=0.17 \mathrm{~m}$; length $a=2.00 \mathrm{~m}$; wideness $b=1.50 \mathrm{~m}$; protective layer thickness $h_{p l}(0.03 \mathrm{~m}$ on the top, $0.04 \mathrm{~m}$ on the bottom); reinforcement rod diameter $d=0.010 \mathrm{~m}$. The number of reinforcing rods in the upper belt: along $x=11$ pieces every $0.14 \mathrm{~m}$; along $y=11$ pieces every $0.19 \mathrm{~m}$; in the lower belt: along $x=15$ pieces every $0.1 \mathrm{~m}$; along $y=16$ pieces every $0.125 \mathrm{~m}$.

The elasticity modulus of the concrete under tension: the unaffected by the chlorides concrete $E_{10}=25091 \mathrm{MPa}$; the affected by the chlorides one $-E_{1}=13200 \mathrm{MPa}$; coefficient $k_{1}=11891 \mathrm{MPa}$. Under compression: the unaffected by the chlorides concrete $E_{20}=37297 \mathrm{MPa}$; affected by the chlorides one $-E_{2}=28989 \mathrm{MPa}$; coefficient $k_{2}=8308 \mathrm{MPa}$; Poisson's ratio for the concrete under the tension and compression is 0.2 . The reinforcement elasticity modulus $E_{r}=180000 \mathrm{MPa}$. The reinforcement yield strength $\sigma_{y}=450 \mathrm{MPa}$. Poisson's ratio for the reinforcement is 0.3 .

The parameters of the elastic base are as follows: base soil - silt loam, the soil elastic modulus is $82 \mathrm{MPa}$, Poisson's ratio for the soil is 0.35 . Parameters of the reinforcement corrosion wear equation: $\delta_{0}=3.06 \cdot 10^{-3} \mathrm{~m}, T=2414$ days. Parameters of the motion equation of the concrete chloride corrosion front: $\alpha=8.26 \cdot 10^{-4} \mathrm{~m} / \mathrm{day}^{0.5}$, grid spacing is $32 \times 32 \times 10$.

Firstly, the analysis was carried out for $0.2 \mathrm{MPa}$ loading level without the chlorides action.

Fig. 1, $a$ and $b$ show the bending diagrams for the rigidly clamped plate along its contour at the moment $t=0$ (line 1 ), $t=10000$ days (line 2 ) and $t=25840$ days (line 3 ) for the main cross-sections.

a)

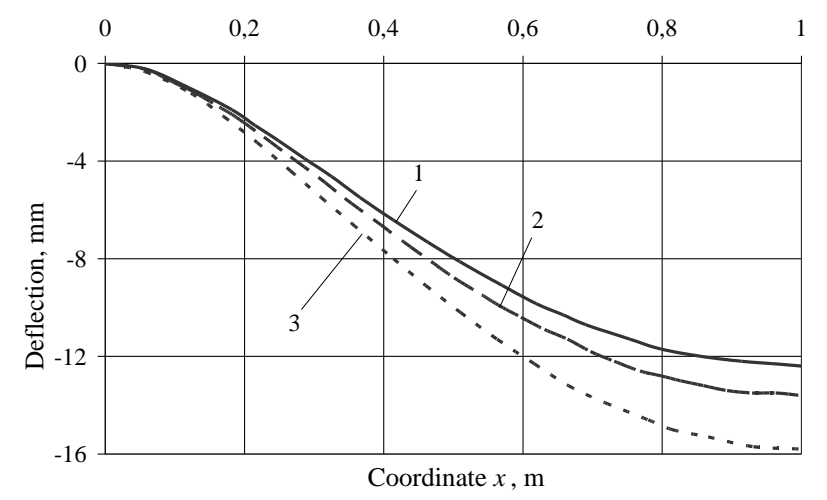

b)

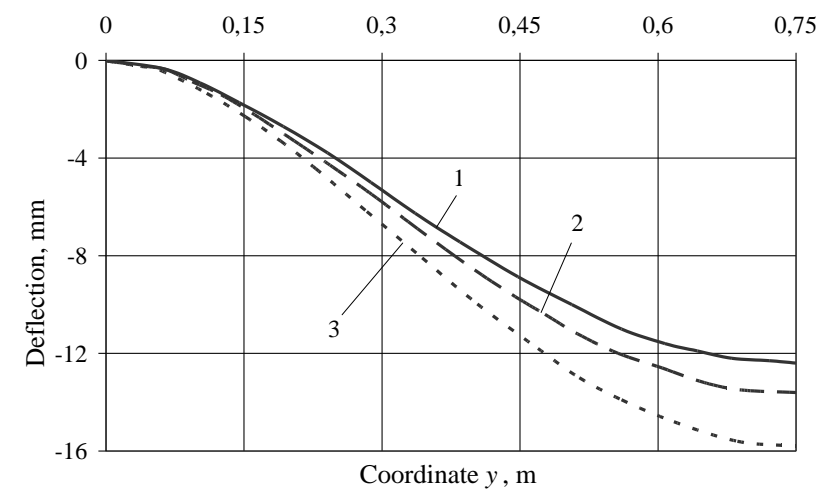

Figure 1. Deflections along the $x$ axis (a), the $y$ axis (b) for the rigidly clamped plate without the chlorides action at $1-t=0,2-t=10000$ days, $3-t=25840$ days.

Then the analysis was carried out at a $0.2 \mathrm{MPa}$ loading level but under the chlorides action. Fig. $2 a$ and $b$ show the bending diagrams for the rigidly clamped plate along its contour at the same time moments. The figures show that the 
presence of chloride corrosion leads to a redistribution of stresses and deformations in the construction material in comparison with the destruction observed due to the reinforced concrete properties change caused by a change in stiffness characteristics from equation (1).
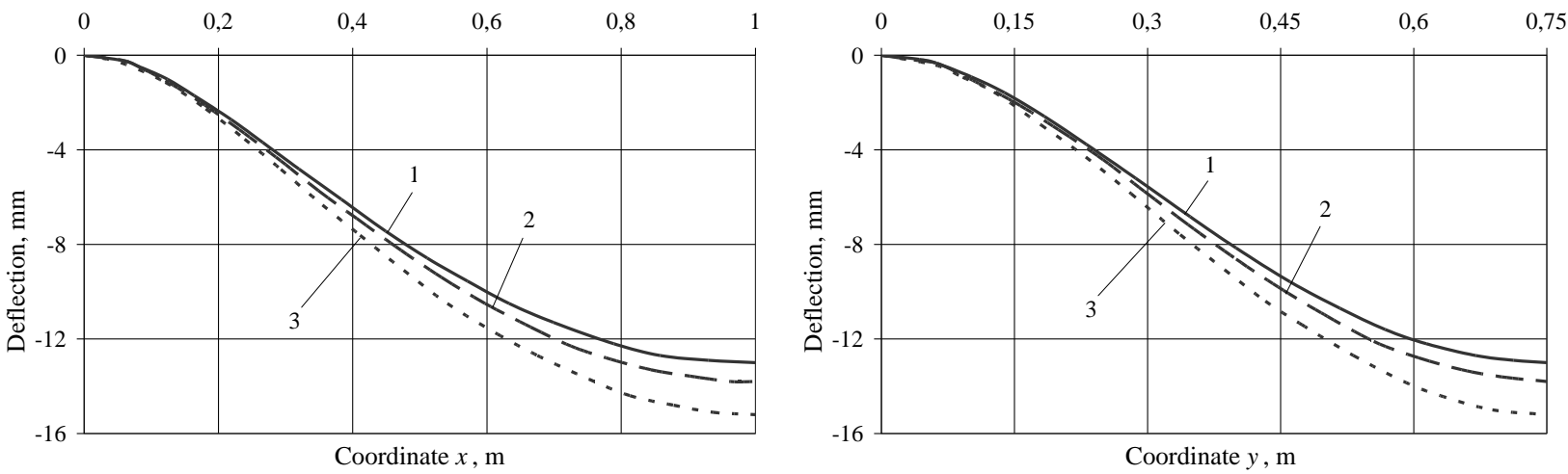

Figure 2. Deflections along the $x$ axis (a), the $y$ axis (b) for the rigidly clamped plate under the chlorides action at $1-t=0,2-t=10000$ days, $3-t=25840$ days.

Fig. 3 shows the calculated curves of the plate durability without (line 1) and under (line 2) the chlorides action. These curves show the values of the external distributed load intensity for the structure strength insurance during a time period plotted along the abscissa axis. As it is observed, there are two sections on the diagrams - rectilinear and hyperbolic. In the first section, the destruction of the plate occurs due to the deformations limit level achievement, and in the second it takes place because of the damage parameter $L$ limit value. The action of chlorides leads to a significant decrease in the structure durability.

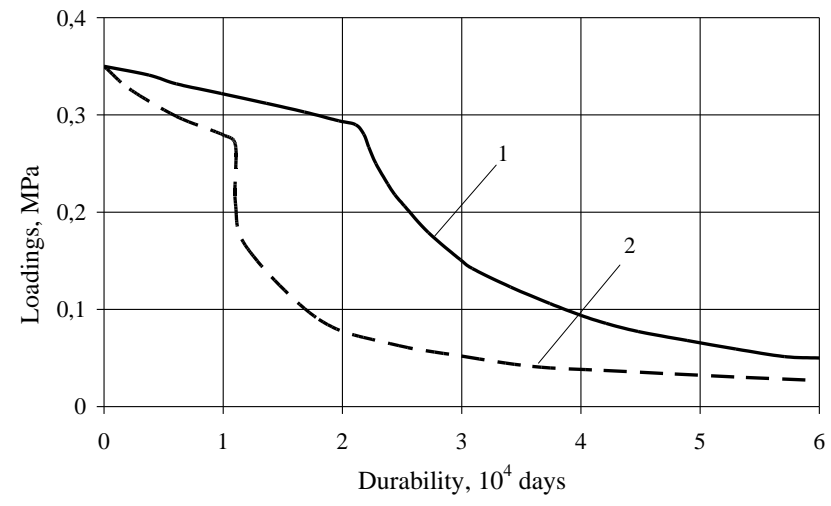

Figure 3. Plate durability without (line 1) and under (line 2) the chlorides action.

\section{CONCLUSIONS}

The performed investigations showed that the elastically-based plate calculating method based on the combined use of parameters successive perturbations and grids methods allows to obtain real durability values for the structures under the chloride corrosion action.

Durability calculation for the considered construction design demonstrated that the presence of chloride corrosion can lead to a reduction in the permissible load on it by 4 times or more. This fact should be taken into account during the design and operation processes of structures in agricultural production.

\section{REFERENCES}

1. Alekseev, S.N., Ivanov, F.M., Modry, S., Schissl, P. 1990. Durability of reinforced concrete in corrosive environments, Stroyizdat, Moscow (in Russian).

2. Biondini, F., Bontempi, F., Frangopol, D. M., Malerba, P. G. 2004. Cellular Automata Approach to Durability Analysis of Concrete Structures in Aggressive Environments. Journal of Structural Engineering ASCE. Vol. 130, Iss. 11, pp. $1724-1737$. http://doi.org/10.1061/(ASCE)0733-9445(2004)130:11(1724)

3. Chen, D., Mahadevan, S. 2008.Chloride-induced reinforcement corrosion and concrete cracking simulation. Cement and Concrete Composites. Vol. 30, Iss. 3, pp. 227-238. http://doi.org/10.1016/j.cemconcomp.2006.10.007

4. Frangopol, D.M., Kallen, M.-J., van Noortwijk. J.M. 2004. Probabilistic models for life-cycle performance of deteriorating structures: review and future directions. Progress in Structural Engineering and Materials. Vol. 6, Iss. 4, pp. 197-212. http://doi.org/10.1002/pse.180 
5. Frangopol, D.M., Lin, K.Y., Estes, A. 1997. Reliability of reinforced concrete girders under corrosion attack. Journal of Structural Engineering ASCE. Vol. 123, Iss. 3, pp. 286-297. http://doi.org/10.1061/(ASCE)0733-9445(1997)123:3(286)

6. Guzeev, E.A., Bondarenko, V.M., Savitsky, N.V. 1984. Integral method for assessing the stress-strain state of reinforced concrete elements under the influence of an aggressive medium and a load. Proceedings of NIIZhB, Stroyizdat, Moscow, pp. 20-27 [In Russian].

7. Karpenko, N.I. 1996. General Models of Reinforced Concrete Mechanics, Stroyizdat, Moscow [In Russian].

8. Kong, J.S., Ababneh, A.N., Frangopol, D.M., Xi, Y. 2002. Reliability analysis of chloride penetration in saturated concrete. Probabilistic Engineering Mechanics. Vol. 17, Iss. 3, pp. 305-315. http://doi.org/10.1016/S0266-8920(02)00014-0

9. Müller, K.F. 1985. The possibility of evolving a theory for predicting the service life of reinforced concrete structures. Materials and Structures. Vol. 18, Iss. 6, pp. 463-472. http://doi.org/10.1007/BF02498752

10. Neville, A. 1995. Chloride attack of reinforced concrete: an overview. Materials and Structures. Vol. 28 , pp. 63-70. http://doi.org/10.1007/BF02473172

11. Ovchinnikov, I.G., Ratkin, V.V., Garibov, R.B. 2002. The efficiency of steel-reinforced concrete structural elements under conditions of exposure to chloride-containing media, Saratov State University, Saratov [In Russian].

12. Ovchinnikov, I.I., Naumova, G.A. 2007. Accumulation of damages in rod and plate reinforced structures interacting with aggressive media, Volgograd State University of Architecture and Civil Engineering, Volgograd [In Russian].

13. Ovchinnikov, I.I., Ovchinnikov, I.G. 2014. Identification and verification of corrosion and deformation processes models, Saratov State Technical University, Saratov [In Russian].

14. Parant, E., Rossi, P., Le Maou, F. 2007. Durability of a multiscale fibre reinforced cement composite in aggressive environment under service load. Cement and Concrete Research. Vol. 37, Iss. 7, pp. 1106-1114. http://doi.org/10.1016/j.cemconres.2006.02.021

15. Petrov, V.V., Ovchinnikov, I.G., Shikhov, Yu.M. 1987. Calculation of structural elements interacting with an aggressive environment, Saratov State University, Saratov [In Russian].

16. Polak, A.F. 1983. Calculation of reinforced concrete structures durability, Ufa Oil Institute, Ufa [In Russian].

17. Popesko, A.I. 1996. Performance of reinforced concrete structures that are susceptible to corrosion, Saint Petersburg State University of Architecture and Civil Engineering, Saint Petersburg.

18. Šadzevičius, R., Sankauskienè, T., Milius, P. 2015. Comparison of concrete compressive strength values obtained using rebound hammer and drilled core specimens. Proceedings of the International Scientific Conference "Rural Development 2015 ", 6 p. http://doi.org/10.15544/RD.2015.011

19. Saetta, A., Scotta, R., Vitaliani, R. 1999. Coupled Environmental-Mechanical Damage Model of RC Structures. Journal of Engineering Mechanics ASCE. Vol. 125, Iss. 8, pp. 930-940. http://doi.org/10.1061/(ASCE)0733-9399(1999)125:8(930)

20. Solomatov, V.I., Selyaev, V.P. 1987. Chemical Resistance of Composite Building Materials. Stroyizdat, Moscow [In Russian].

21. Wright, J.R., Frohnsdorff, G. 1985. Durability of building materials: Durability research in the United States and the influence of RILEM on durability research. Materials and Structures. Vol. 18, Iss. 3, pp. 205-214. http://doi.org/10.1007/BF02472971

22. Zhong, J., Gardoni, P., Rosowsky, D. 2010. Stiffness Degradation and Time to Cracking of Cover Concrete in Reinforced Concrete Structures Subject to Corrosion. Journal of Engineering Mechanics ASCE. Vol. 136, Iss. 2, pp. $209-219$. http://doi.org/10.1061/(ASCE)EM.1943-7889.0000074

23. Zhou, Y., Gencturk, B., Willam, K., Attar, A. 2015. Carbonation-Induced and Chloride-Induced Corrosion in Reinforced Concrete Structures. Journal of Materials in Civil Engineering. Vol. 27, Iss. 9. http://doi.org/10.1061/(ASCE)MT.1943-5533.0001209 